\title{
Enhancement of Time-domain Optical Coherence Tomography Images Using Stationary Wavelet Transform
}

\author{
Manting Luo1,a, Tengfei You1,b,Qiukun Zhang1,c, Jianfeng \\ Zhong1,d, Shuncong Zhong1,2,e* \\ 1Laboratory of Optics, Terahertz and Non-destructive Testing \& \\ Evaluation, School of Mechanical Engineering and Automation, Fuzhou \\ University, Fuzhou 350108, China; \\ 2Fujian Key Laboratory of Medical Instrument and Pharmaceutical \\ Technology, Fuzhou 350108, P.R. China \\ a289251753@qq.com,b460992456@qq.com,c331929147@qq.com, \\ d1083671123@qq.com,ezhongshuncong@hotmail.com(corresponding \\ author)
}

\begin{abstract}
Stationary wavelet transform (SWT) was proposed to enhance time-domain optical coherence tomography (OCT) images of multilayered thin-film structures. Multi-level SWT decomposition of OCT images was conducted and the SWT detail coefficients could be employed for feature extraction of multilayered structures' interface. The experimental results demonstrated that SWT-based technique could achieve better OCT image quality than traditional OCT imaging, making it attractive for non-medical applications.
\end{abstract}

Keywords: time-domain optical coherence tomography; Stationary Wavelet Transform;balanced detection;thinfilmcharacterization;image enhancement

\section{Introduction}

Optical coherence tomography (OCT) is a novel imaging technique proposed by Huang[1]. Due to its excellent optical nondestructive slicing ability and high resolution,OCT has been widely used in the eye structure imaging of the human, dental detection and any other biomedical field. In recent years, its outstanding detection capability also extends to the industrial use such as jade structure analysis, thin film delamination, Pharmaceutical tablet characterization [2].Time-domain optical coherence tomography system based on the balanced detection, overcomes the influence of self-coherent noise and complex conjugate mirror image of frequency domain, achieves a higher signal noise ratio and deeper detection depth. However, during the imaging process, as the presence of light source noise, photoelectric detector noise, scanning module noise and beat noise components of incoherent light reflect from sample, output 
of OCT image exists the speckle, therefore, various image processing method based on OCT system emerging [3]. Time domain optical coherence tomography system usually employs short -Time Fouriertransform (STFT) to complete the image reconstruction. STFT algorithm is convenient, but its time-frequency resolution for any frequency is fixed, leading to the problems in obtaining the better denoising results in interference signal demodulation[4]. Wavelet analysis is the inheritance and development of the short-time Fourier transform,it has gradually grown into a very useful tool in the field of mechanical structure health monitoring, and been aptly called as "mathematical microscope".With the multi resolutioncharacteristics in time-frequency domain,wavelet transform can carry out local analysis and signal local singularities feature extraction flexible at the same time. By using the wavelet transform,we can conduct the data compression, data fusion, image enhancement and denoising simply[5].Stationary wavelet transform (SWT) is developed in basis of orthogonal wavelet, proposed by Nason and Silverman in 1995[6], it does not exist a Gibbs shock problem when signal reconstruction. SWT was applied in the time domain optical coherence tomography system in the present work.For SWT, OCT image is decomposed into approximation and detail coefficients. The low frequency information of image is in theapproximation coefficient whilst high frequency information is included in the detail coefficients.

\section{SWT-basedtime-domain optical coherence tomography}

The core of OCTsystem is a Michelson interferometer[7]. The light emitted by the light source propagated through 50:50 beam splitter and been splitted into two beams, respectively propagating into the reference arm(with mirror) and sample arm(with sample). When the reference arm scanning vertically, the sample arm scanning transversely simultaneously, photoelectric detector detected the two beams of light interference intensity signal and the converted themto electrical signals; then signal processing system transformthe two-dimensional scanning information into the two-dimensional image information.

The principle of balanced detection was employedto build OCT system. Owing to the system using light coherenceprinciple to get longitudinal resolution information, so the optical signal arrived at the photoelectric detector follows the typical interference pattern of double beam [8],

$$
I_{\mathrm{P}}(\Delta l)=I_{\mathrm{S}}+I_{\mathrm{R}}+2 \sqrt{I_{S} I_{R}}\left|\tau_{S R}(\Delta l)\right| \bullet \cos \left(k_{0} \Delta l+\alpha_{S R}\right) .
$$

where $\Delta l$ is the path between the signal light and the reference light, $I \mathrm{~s}, I_{\mathrm{R}}$ are the light intensity of two beams of light, respectively. $\tau_{S R}(\Delta l)$ is the normalized complex correlation function of reference light and signal light, $k_{0}$ is light propagation constants, $\alpha_{S R}$ is sample light's initial phase relative to the reference wave.In the balanced detection, average power reachdetector D1 and D2 is considered to be the same,at the same time, interference signal has the phase difference of $\pi$, therefore in the differential amplifier, the bottom signal eliminated [9]. In Eq.(1), the first two 
items are eliminated, the interference signal is enhanced. So, balanced detection onlyretain the interference signal for the follow-up signal process, so the SNR has been improved.

As shown in Fig.1, The light source employed50W tungsten halogen lamps[10].In the system, light propagatedfirst through an oblique placed plastic sheets, with characteristicsof strong transmission, 95\% light continued to travel along a straight line through plastic sheets for reference and sample arms' interference, the interference intensity detected by the photoelectric detector D2; $5 \%$ of the light reflected by the plastic sheet and was led to the D1 detectorandthen was used to balance the DCbackground noise (there provided an adjustable light hole to regulate D1's intensity balance with D2's intensity).The two detectorsmade up a balanced module, the differential signal had been amplified and put intodata acquisition (DAQ) card, finally transferred into the computer for imaging process. Scanning platform of system was conducted byThorlabs MTS25-Z8 25mm compact electric platform whose minimum increment can reach $0.05 \mu \mathrm{m}$, which can maintain high stability during scanning process. Balanced detection module used in the present work wasThorlabs PDB 210A/M, which has two large area silicon sensorsand thedetection zoneranges from 320 to $1060 \mathrm{~nm}$. DAQ card employed NI USB-6009 with 14 bitdifferential AI resolution and the maximum sampling rate of $48 \mathrm{kS} / \mathrm{s}$. The center wavelength is $700 \mathrm{~nm}$ and the spectrum width is $236 \mathrm{~nm}$ of our system. The theoretical OCT resolution can be obtained by $(2 \ln 2) / \pi \lambda_{0}^{2} / \Delta \lambda=0.9 \mu \mathrm{m}_{[11]}$.

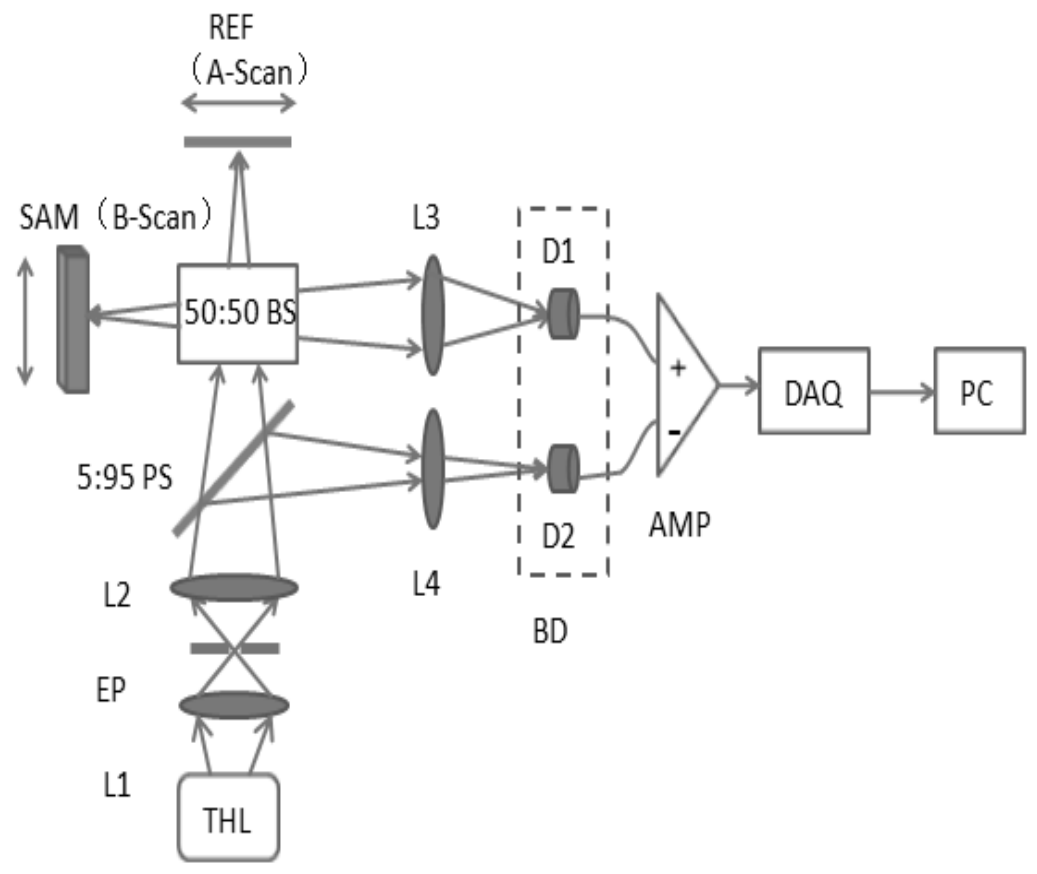


Fig. 1. Schematic diagram of the time-domain OCT experimental setup: THL: Tungsten Halogen Lamp; EP: Entrance pupil; L1/L2: Lens; REF: reference arm; BS, beam-splitter; SAM: samples;
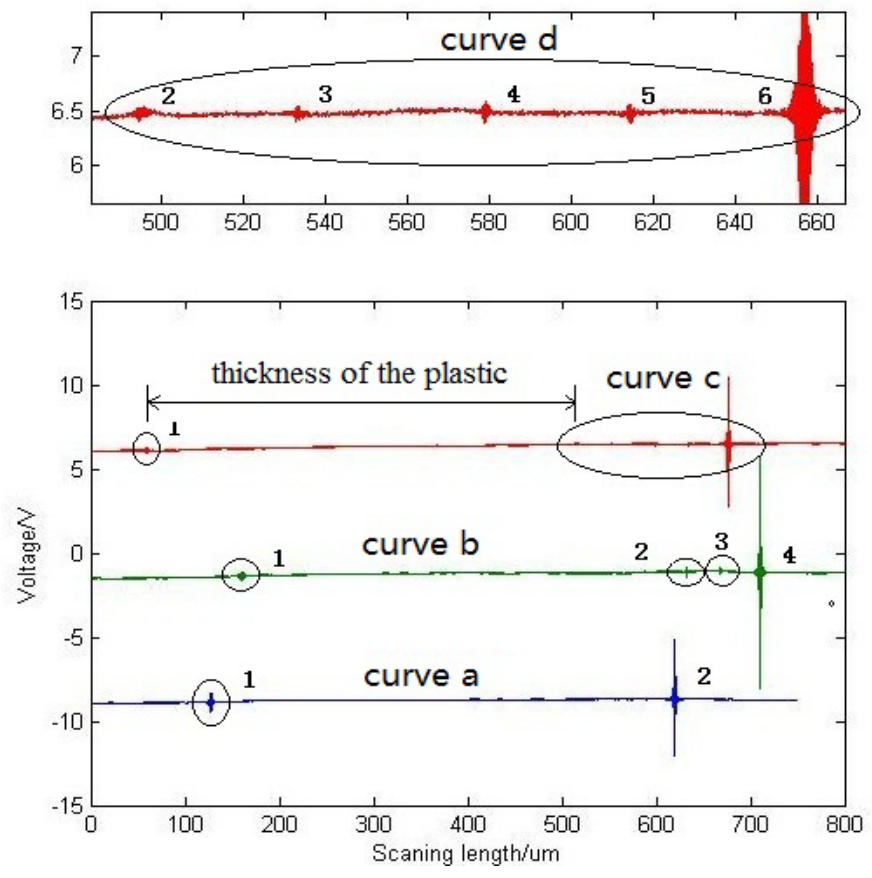

Fig.2. One dimensional time-domaininterferogram of multiple samples; (a) 320um plastic sheet; (b) a plastic sheet pasted with a transparent adhesive tape; (c) a plastic sheet pasted with two transparent tape ; (d)zoomed figure of curve (c).

\section{Results and Discussions}

Fig.2 shows one dimensional time-domaininterferogram of multiple samples: (a) plastic sheet; (b) a plastic sheet pasted with a transparent adhesive tape; (c) a plastic sheet pasted with two transparent tapes.The figure demonstrated the capability of the developed OCT system in characterization of multi-layered thin-film structures.

In the OCT image, pixel gray level is in proportion to photocurrent, the emergence of speckle will make some pixel random variable of image becomes dark, will cause a lot ofnoise,making the details of the image can't be clearly seen and then reducing the quality of image, as shown in Figure 3(a), the original OCT image. There is too much noise in the OCT image, the useful structural information has been hided, and so SWT algorithm was used to extract more significant interface delamination of collected data.This OCT imagewasSWT 
decomposed into the approximation coefficients and detail coefficients. The low frequency information of image is in theapproximation coefficient whilst high frequency information is included in the detail coefficients [14]. Fig.3 (b) shows the detail coefficients of OCT image by two-level SWT decomposition. We could find that the interfaces of the multi-layered thin-film structure are shown clearly and therefore SWT has could be used to enhance the quality of OCT images. In order to investigate the effect of number of SWT decomposition level on the OCT image quality. SWT decomposition level of 3 and 4 was applied to the original OCT image. Fig.3 (c) and (d), respectively, show the detail coefficients of OCT image by three-level and four-level SWT decompositions. From these two figures, we could also identify the interfaces of the multilayered structures, however, the images start to be unclear if SWT decomposition level of 4 due to the edge effect. Furthermore, if SWT decomposition level of 5, the image (no shown in the paper due to page limit) got worse.We found thatthe severity of the edge effect increases with the decomposition levels.
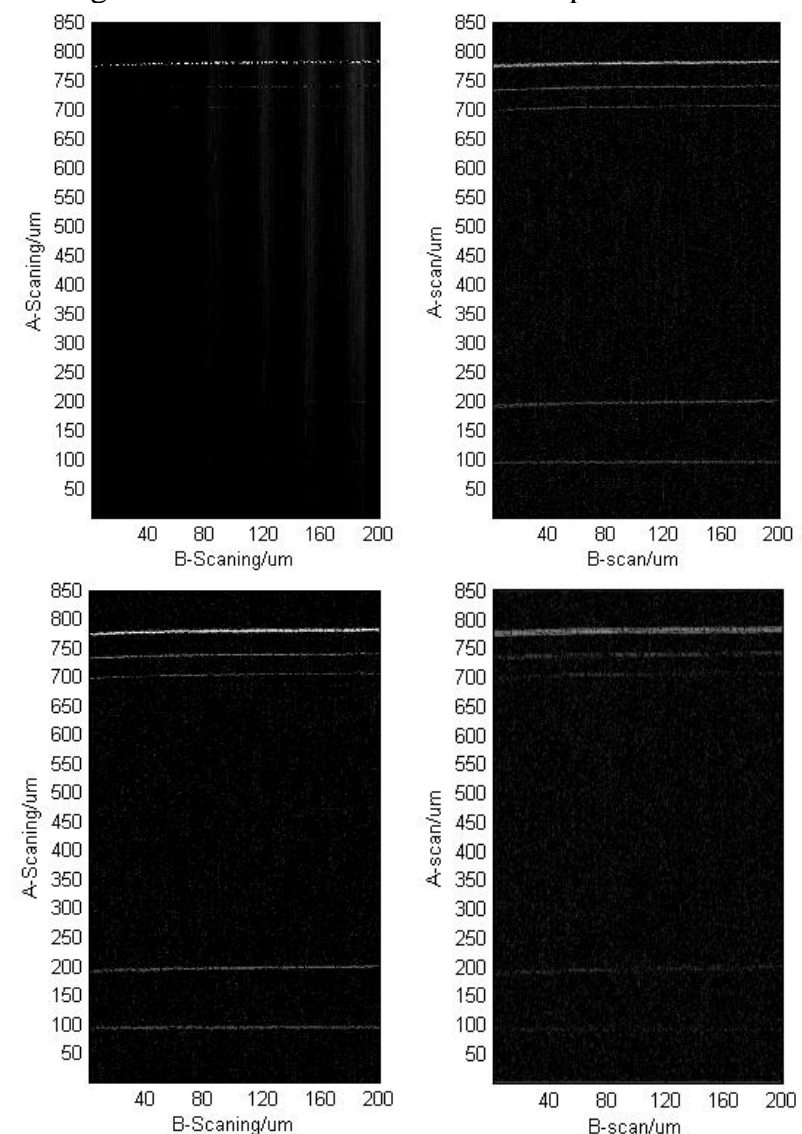

Fig.3.(a) Original OCT Image; (b) the detail coefficients of OCT image by two-level SWT decomposition; (c) the detail coefficients of OCT image by 
three-level SWT decomposition;.(d)the detail coefficients of OCT image by four-level SWT decomposition.

From the results above,we need to attention to the existence of the edge effect while using the SWT in OCT images. The severity of edge effect increases with the decomposition levels. It needs toextentthe original data using some signal extension algorithms.In the proposed SWT-based OCTimaging, only synthetically considering the edge effect with the effect of image wavelet decomposition during selecting thesuitable wavelet levelscan we get a best image quality.

\section{Conclusions}

An optical coherence tomography system with a resolution of $0.9 \mu \mathrm{m}$ has been developed.The system had excellent performance and its depth resolution was less affected by sample scattering[15]. In addition, Stationary wavelet transform (SWT) was proposed to enhance time-domain optical coherence tomography (OCT) images of multilayered thin-film structures. Multi-level SWT decomposition of OCT images was conducted, from which the SWT detail coefficients could be employed for feature extraction of multilayered structures' interface. The experimental results demonstrated that SWT-based technique could achieve better OCT image quality than traditional OCT imaging, making it attractive for non-medical applications, such as nondestructive detection of composite material and other fiber material. However, compared with the frequency-domain OCT, the speed of time-domain OCT system still needs to be considered.

\section{Acknowledgement}

We gratefully acknowledge support from National Natural Science Foundation of China, Natural Science Foundation of Fujian Province,Training Program of Fujian Excellent Talents in University, Specialized Research Fund for the Doctoral Program of Higher Education, Ministry of Education, P.R. China (20133514110008), Ministry of Health, P.R. China (WKJ-FJ-27), Fujian Provincial Bureau of Quality and TechnicalSupervision (FJQI2013095 ,FJQI2012024) and The State Quality Inspection Administration of science and technology project (2011QK216).

\section{References}

[1].Huang D, Swanson EA, Lin CP, Schuman JS, Stinson WG,Chang W, Hee MR, Flotte T, Gregory K, Puliafito CA et al(1991) Optical coherence tomography. Science 254(5035):1178-118

[2].R.K. May, K. Su, L. Han, ShuncongZhong, J. A. Elliott, L. F. Gladden, M. Evans, Y. C. Shen, and J. A. Zeitler, Hardness and Density Distributions of Pharmaceutical Tablets Measured by Terahertz Pulsed Imaging, Journal of Pharmaceutical Sciences, 2013 (DOI: 10.1002/jps.23560) 
[3].Larkin KG. 1996. Efficient nonlinear algorithm for envelope detection in white light interferometry. J Opt Soc Am A 13:832-843.

[4].Tay, C.J.,Quan, C.,Sun, W.,He, X.Y. Demodulation of a single interferogram based on continuous wavelet transform and phase derivative. Optics Communications 2007;2(280):327-336.

[5].ChitchianS;FiddyMA;Fried NM. Denoising during optical coherence tomography of the prostate nerves via wavelet shrinkage using dual-tree complex wavelet transform. Journal of Biomedical Optics 2009;1(14):014031.

[6].Nason G P ,Sliverman B W .The Stationary Wavelet Transform and Some Statistical Application in Wavelet and Statistics. 1996

[7].Adolf Friedrich Fercher. Optical coherence tomography - development, principles,applications. ScienceDirect 2009;11(02):1-27.

[8].XianongZhu;YanmeiLiang;YouxinMao;YaqingJia;Yiheng Liu and Guoguang Mu. Analyses and calculations of noise in optical coherence tomography systems. Frontiers of Optoelectronics in China 2008;3-4(1):247-257.

[9].Cooper, Merlin;Söller, Christoph;Smith, Brian J. High-stability time-domain balanced homodyne detector for ultrafast optical pulse applications. Journal of Modern Optics 2013;8(60):611-616.

[10].ShuncongZhong,Yaochun Shen. Real-time monitoring of structural vibration using spectral-domain optical coherence tomography. Optics and Lasers in Engineering 2011;49:127-131.

[11].ShuncongZhong,Yao-Chun Shen,Louise Ho,Robert K. May, et al. Non-destructive quantification of pharmaceutical tablet coatings using terahertz pulsed imaging and optical coherence tomography. Optics and Lasers in Engineering 2011;49:361-365.

[12].Chen Li ,J. Axel Zeitler, Yue Dong, Yao-Chun Shen. Non-Destructive Evaluation of Polymer Coating Structures on Pharmaceutical Pellets Using Full-Field Optical Coherence Tomography. Journal of Pharmaceutical Sciences 2013;10.

[13].ShuncongZhong, S. OlutundeOyadiji. Crack detection in simply supported beams without baseline modal parameters by stationary wavelet transform. Mechanical Systems and Signal Processing 2007; 21:1853-1884.

[14].Xin Feng,XiaotanZhang,ChangsenSun,MohammadhoseinMotamedi,Farhad Ansari . Stationary Wavelet Transform Method for Distributed Detection of Damage by Fiber-Optic Sensors. Journal of Engineering Mechanics2014;04(140) 
[15].Dubois A, Boccara AC. 2008. Full-field optical coherence tomography. In Optical coherence tomography; Drexler W, Fujimoto JG, editors.Heidelberg, Germany: Springer, pp 565-591. 